Original Research Paper

\title{
$E$-Saddle Point Criteria for $E$-differentiable Vector Optimization Problems with Inequality and Equality Constraints
}

\author{
${ }^{1}$ Tadeusz Antczak and ${ }^{2}$ Najeeb Abdulaleem \\ ${ }^{1}$ Faculty of Mathematics and Computer Science, University of Łódź, Banacha 22, 90-238 Lódź, Poland \\ ${ }^{2}$ Department of Mathematics, Hadhramout University, P.O. BOX: (50511-50512), Al-Mahrah, Yemen
}

\author{
Article history \\ Received: 13-02-2019 \\ Revised: 08-04-2019 \\ Accepted: 10-05-2019 \\ Corresponding Author: \\ Najeeb Abdulaleem \\ Department of Mathematics, \\ Hadhramout University, P.O. \\ BOX: (50511-50512), \\ Al-Mahrah, Yemen \\ Email: nabbas985@gmail.com
}

\begin{abstract}
In this study, the class of $E$-differentiable vector optimization problems with both inequality and equality constraints is considered. For such (not necessarily) differentiable multicriteria optimization problems, two $E$-Lagrange functions and their $E$-saddle points are defined. Then the so-called $E$-saddle point criteria are established for such (not necessarily) differentiable multiobjective programming problems with $E$ differentiable $E$-convex functions.
\end{abstract}

Keywords: $E$-differentiable Vector Optimization Problem, $E$-saddle Point, E-Lagrange Function, E-saddle Point Criteria, E-convex Function

\section{Introduction}

In recent years, saddle point criteria for vector optimization problems, also called multiobjective programming problems or multicriteria optimization problems, have attracted much interest. There are several reasons for studying the characterization of a (weak) Pareto solution for multicriteria optimization problems via the saddle point criteria. But probably the most important one is related to the result that a saddle point of the Lagrange function is always a global optimal solution of a constrained optimization problem. Because of the importance of this result, for example, in optimization theory and economics, therefore, many authors have analyzed and studied theory of saddle point criteria for nonconvex multiobjective programming problems (see, for instance, (Adán and Novo, 2005; Antczak, 2003; 2005; 2015; Bhatia, 2008; Bigi, 2001; Craven, 1990; Ehrgott and Wiecek, 2005; Jiang and $\mathrm{Xu}, 2010$; Kuk et al., 1998; Kumar and Garg, 2015; Maciel et al., 2016; Mishra and Giorgi, 2008; Li and Wang, 1994; Van Rooyen et al., 1994; Tanaka, 1990; 1994; Tanino, 1982; Vályi, 1987; Varalakshmi et al., 2010; Yan and Li, 2004; Zeng, 2017). Taninio (1982) proved that solutions of multicriteria optimization problems and corresponding multiplier vectors are saddle points of vector-valued Lagrange functions. Used a scalarization method, Tanaka (1990) characterized generalized saddle points of vector-valued Lagrangians. Li and Wang (1994) derived several conditions for the existence of a Lagrange multiplier or a weak saddle point of vector optimization problems and proved relationships between them. Bigi (2001) proved criteria for saddle points of nondifferentiable multiobjective programming problems under convexity hypotheses. Ehrgott and Wiecek (2005) studied relations between saddle points and Pareto points for both convex and nonconvex vector optimization problems used scalarizations. Antczak (2003; 2005) proved saddle point criteria for the considered multiobjective programming problem by using the introduced nonlinear approximation methods. Bhatia (2008) established the equivalence between a mixed saddle point of order $m$ defined for the vector-valued partial Lagrange function and higher order minima of the considered nondifferentiable multiobjective programming problem. Kumar and Garg (2015) introduced the definition of a socalled mixed saddle point of a vector-valued Lagrange function defined for the considered nonsmooth multicriteria optimization problem and proved the equivalence between a Pareto solution and a mixed saddle point under the concept of generalized $(V, \rho)$-invexity. Recently, under generalized notions of convexity, Maciel et al. (2016) investigated the relations between a Fritz John critical point of the considered differentiable multiobjective programming problem and a saddle point of the vector-valued Lagrange function.

One of generalized convexity notions introduced into optimization theory is the concept of $E$-convexity which was defined by Youness (1999). The concept of $E$ convexity is based on the effecting of an operator 
$E: R^{n} \rightarrow R^{n}$ on the sets and the domain on which functions are defined. Recently, the definition of an $E$ differentiable function was introduced by Megahed et al. (2013) in which, based on the effect of an operator $E: R^{n} \rightarrow R^{n}$, a (not necessarily) differentiable function is transformed to a differentiable function (in the usual sense).

Up to now, the most of papers dealing with the characterization of Pareto optimality by saddle point criteria is restricted to differentiable multiobjective programming problems. Therefore, in the present paper, we consider the class of $E$-differentiable multiobjective programming problems with both inequality and equality constraints in which the functions involved are (not necessarily) differentiable. For such (not necessarily) differentiable vector optimization problems involving $E$ differentiable functions, characterizations of their saddle points are presented. In the used approach, for the original E-differentiable multicriteria optimization problem, its related $E$-vector optimization problem is constructed in which the involved functions are differentiable (in the usual sense). Then, so-called scalar and vector-valued $E$-Lagrange functions and their $E$ saddle points are defined for the considered multiobjective programming problem. Further, the socalled $E$-saddle point criteria are established for such (not necessarily) differentiable multiobjective programming problems under $E$-convexity hypotheses. Then, the equivalence is proved between (weak) $E$ Pareto solutions and $E$-saddle points of scalar and vectorial $E$-Lagrange functions defined in the considered $E$-differentiable multiobjective programming problem with $E$-convex functions via the similar result established for its associated $E$-vector optimization problem. Hence, tools of differentiable analysis are used in proving saddle point criteria for (not necessarily) differentiable multiobjective programming problems.

\section{Preliminaries}

In this section, we provide some notations and results that we shall use in the sequel.

Let $R^{n}$ be the n-dimensional Euclidean space and $R_{+}^{n}$ be its nonnegative orthant. The following convention for equalities and inequalities will be used in the paper. Namely, for any vectors $x=\left(x_{1}, x_{2}, \ldots, x_{n}\right)^{T}$ and $y=\left(y_{1}\right.$, $\left.y_{2}, \ldots, y_{n}\right)^{T}$ in $R^{n}$, we define $x=y$ if and only if $x_{i}=y_{i}$ for all $i=1,2, \ldots, n ; x \geqq y$ if and only if $x_{i} \geqq y_{i}$ for all $i=$ $1,2, \ldots, n ; x \geqq y$ if and only if $x_{i}>y_{i}$ for all $i=1,2, \ldots, n$; $x \geq y$ if and only if $x \geqq y$ and $x \neq y$.

Definition 1 (Youness, 1999)

A set $M \subseteq R^{n}$ is said to be $E$-convex (with respect to an operator $E: R^{n} \rightarrow R^{n}$ ) if and only if the following relation:

$$
E(u)+\lambda(E(x)-E(u)) \in M
$$

holds for all $x, u \in M$ and any $\lambda \in[0,1]$.

\section{Definition 2 (Youness, 1999)}

Let $E: R^{n} \rightarrow R^{n}$ and $M$ be a nonempty $E$-convex subset of $R^{n} . f: M \rightarrow R$ is said an $E$-convex function on $M$ if and only if the inequality:

$$
\begin{aligned}
f(\lambda E(x)+(1-\lambda) E(u)) \leqq \\
\lambda f(E(x))+(1-\lambda) f(E(u))
\end{aligned}
$$

is satisfied for all $x, u \in M$ and any $\lambda \in[0,1]$.

\section{Definition 3 (Megahed et al., 2013)}

Let $E: R^{n} \rightarrow R^{n}, M$ be an open $E$-convex set, $f: M \rightarrow$ $R$ be a (not necessarily) differentiable function at a given point $u$. It is said that $f$ is an $E$-differentiable function at $u$ if and only if $f \circ E$ is a differentiable function at $u$ (in the usual sense) and, moreover:

$$
\begin{gathered}
(f \circ E)(x)=(f \circ E)(u)+\nabla(f \circ E)(u)(x-u)+ \\
\theta(u, x-u)\|x-u\|,
\end{gathered}
$$

where, $\theta(u, x-u) \rightarrow 0$ as $x \rightarrow u$.

\section{Proposition 4}

Let $E: R^{n} \rightarrow R^{n}, M$ be an $E$-convex subset of $R^{n}$ and $f: R^{n} \rightarrow \mathrm{R}$ be an $E$-convex (a strictly $E$-convex) function on $M$ and $u \in M$. Moreover, we assume that the function $f$ is $E$-differentiable at $u$. Then, the inequality:

$$
f(E(x))-f(E(u)) \geqq \nabla f(E(u))(E(x)-E(u)), \quad(>)
$$

holds for all $x \in M,(E(x)) \neq(E(u))$.

In the paper, we consider the following (not necessarily differentiable) vector optimization problem (VP) with both inequality and equality constraints defined as follows:

$$
\begin{gathered}
\text { minimize } f(x)=\left(f_{1}(x), \ldots, f_{p}(x)\right) \\
\text { subject to } g_{j}(x) \leqq 0, \quad j \in J=\{1, \ldots, m\}, \quad(V P) \\
h_{t}(x)=0, \quad t \in T=\{1, \ldots, q\}, \\
x \in R^{n},
\end{gathered}
$$

where, $f_{i}: R^{n} \rightarrow R, i \in I=\{1, \ldots, p\}, g_{j}: R^{n} \rightarrow R, j \in J$, $h_{i}: R^{n} \rightarrow R, t \in T$, are functions defined on $R^{n}$. Let $\Omega$ denote the set of all feasible solutions of the problem (VP), that is, the set: 


$$
\Omega:=\left\{x \in R^{n}: g_{j}(x) \leqq 0, j \in J, h_{t}(x)=0, t \in T\right\} .
$$

Further, the set of inequality constraint indices that are active at a feasible solution $\bar{x}$ is denoted by $J(\bar{x})$ and it is defined by $J(\bar{x})=\left\{j \in J: g_{j}(\bar{x})=0\right\}$.

For such multicriteria optimization problems, the key optimality concept is that of a Pareto (a weak Pareto) solution which is defined as follows.

\section{Definition 5}

$\bar{x} \in \Omega$ is said to be a weak Pareto solution (a weakly efficient solution) of the problem (VP) if and only if there is no another feasible point $x$ such that:

$$
f(x)<f(\bar{x}) .
$$

\section{Definition 6}

$\bar{x} \in \Omega$ is said to be a Pareto solution (an efficient solution) of the problem (VP) if and only if there is no another feasible point $x$ such that:

$$
f(x) \leq f(\bar{x}) .
$$

Let $E: R^{n} \rightarrow R^{n}$ be a given one-to-one and onto operator. Throughout the paper, we shall assume that the functions involved in the vector optimization problem (VP) are $E$-differentiable at any its feasible solution.

Now, for the original vector optimization problem (VP), we define its associated differentiable multicriteria optimization problem as follows:

$$
\begin{array}{cc}
\operatorname{minimize} & f(E(x))=\left(f_{1}(E(x)), \ldots, f_{p}(E(x))\right) \\
\text { subject to } & g_{j}(E(x)) \leqq 0, j \in J=\{1, \ldots, m\},\left(\mathrm{VP}_{\mathrm{E}}\right) \\
& h_{t}(E(x))=0, t \in T=\{1, \ldots, q\}, \\
& x \in R^{n} .
\end{array}
$$

We call the multiple objective programming problem $\left(\mathrm{VP}_{\mathrm{E}}\right)$ an $E$-vector optimization problem associated to the original multicriteria optimization problem (VP). Let $\Omega_{E}:=\left\{x \in R^{n}: g_{j}(E(x)) \leqq 0, j \in J, h_{t}(E(x))=0, t \in T\right\}$. be the set of all feasible solutions of the $E$-vector optimization problem $\left(V P_{E}\right)$. Since all functions constituting the considered multiobjective programming problem (VP) are assumed to be Edifferentiable at any its feasible solution, by Definition 3, all functions involved in its associated E-vector optimization problem $\left(V P_{E}\right)$ are differentiable at any its feasible solution (in the usual sense).

Let $J_{E}(x)$ denote the set of all inequality constraint indices that are active at $x \in \Omega_{E}$, that is, $J_{E}(x)=\left\{j \in J: g_{j}(E(x))=0\right\}$.

\section{Lemma 7 (Antczak and Abdulaleem, 2018)}

Let $E: R^{n} \rightarrow R^{n}$ be a one-to-one and onto and $\Omega_{E}:=\left\{x \in R^{n}: g_{j}(E(x)) \leqq 0, j \in J, h_{t}(E(x))=0, t \in T\right\}$. Then $E\left(\Omega_{E}\right)=\Omega$.

Now, we give the relationship between (weak) Pareto solutions in both multiobjective programming problems (VP) and $\left(V P_{E}\right)$ which was established by Antczak and Abdulaleem (2018).

\section{Lemma 8 (Antczak and Abdulaleem, 2018)}

Let $\bar{z} \in \Omega$ be a weak Pareto solution (a Pareto solution) of the considered multicriteria optimization problem (VP). Then, there exists $\bar{x} \in \Omega_{E}$ such that $\bar{z}=E(\bar{x})$ and $\bar{x}$ is a weak Pareto (a Pareto) solution of the $E$-vector optimization problem $\left(V P_{E}\right)$.

\section{Lemma 9 (Antczak and Abdulaleem, 2018)}

Let $\bar{x} \in \Omega_{E}$ be a weak Pareto (Pareto) solution of the $E$-vector optimization problem $\left(V P_{E}\right)$. Then $E(\bar{x})$ is a weak Pareto solution (Pareto solution) of the considered multicriteria programming problem (VP).

\section{Remark 10}

As it follows from Lemma 9, if $\bar{x} \in \Omega_{E}$ is a weak Pareto (Pareto) solution of the $E$-vector optimization problem $\left(V P_{E}\right)$, then $E(\bar{x}) \in \Omega$ is a weak Pareto solution (Pareto solution) of the considered multiobjective programming problem (VP). We call $E(\bar{x})$ a weak $E$-Pareto solution (E-Pareto solution) of the original multiobjective optimization problem (VP).

\section{Theorem 11 (Antczak and Abdulaleem, 2018) (E-Karush-Kuhn-Tucker Necessary Optimality Conditions (E-KKT Conditions, for short) for (VP))}

Let $\bar{x} \in \Omega_{E}$ be a weak Pareto solution of the $E$-vector optimization problem $\left(\mathrm{VP}_{\mathrm{E}}\right.$ ) (and, thus, $E(\bar{x})$ be a weak $E$-Pareto solution of the considered multiobjective programming problem (VP)). Further, assume that the objective functions $f_{i}, i \in I$, the constraint functions $g_{\text {, }}$, $j \in J$ and $h_{t}, t \in T$, are $E$-differentiable at $\bar{x}$ and the E-Abadie Constraint Qualification $\left(\mathrm{ACQ}_{\mathrm{E}}\right)$ is satisfied at $\bar{x}$. Then there exist Lagrange multipliers $\bar{\lambda} \in R^{p}, \bar{\mu} \in R^{m}$ and $\bar{\xi} \in R^{q}$ such that:

$$
\begin{gathered}
\sum_{i=1}^{p} \bar{\lambda}_{i} \nabla f_{i}(E(\bar{x}))+\sum_{j=1}^{m} \bar{\mu}_{j} \nabla g_{j}(E(\bar{x}))+ \\
\sum_{t=1}^{q} \bar{\xi}_{t} \nabla h_{t}(E(\bar{x}))=0,
\end{gathered}
$$

$\bar{\mu}_{j}\left(g_{j} \circ E\right)(\bar{x})=0, j \in J$, 


\section{$\bar{\lambda} \geq 0, \bar{\mu} \geqq 0$.}

We now give the definitions of a Karush-KuhnTucker point for the E-vector optimization problem $\left(\mathrm{VP}_{\mathrm{E}}\right)$ and an E-Karush-Kuhn-Tucker point for the considered multiobjective programming problem (VP).

\section{Definition 12}

We say that $(\bar{x}, \bar{\lambda}, \bar{\mu}, \bar{\xi}) \in \Omega_{E} \times R^{p} \times R^{m} \times R^{s} \quad$ is a Karush-Kuhn-Tucker point (KKT point, for short) of the $E$-vector optimization problem $\left(\mathrm{VP}_{\mathrm{E}}\right)$ if the KarushKuhn-Tucker necessary optimality conditions (4)-(6) are fulfilled at $\bar{x}$ with Lagrange multipliers $\bar{\lambda}, \bar{\mu}, \bar{\xi}$.

\section{Definition 13}

Let $\bar{x}$ be a feasible solution of the $E$-vector optimization problem $\left(\mathrm{VP}_{\mathrm{E}}\right)$. Then $(E(\bar{x}), \bar{\lambda}, \bar{\mu}, \bar{\xi}) \in \Omega_{E} \times R^{p} \times R^{m} \times R^{s}$ is said to be an $E$ Karush-Kuhn-Tucker point ( $E$-KKT point, for short) of the considered multiobjective programming problem (VP) if the $E$-Karush-Kuhn-Tucker necessary optimality conditions (4)-(6) are satisfied at $\bar{x}$ with Lagrange multipliers $\bar{\lambda}, \bar{\mu}, \bar{\xi}$.

\section{Scalar $E$-saddle Point Criteria}

Now, we introduce the definition of the scalar Lagrange function $L_{E}: \Omega_{E} \times R_{+}^{p} \times R_{+}^{m} \times R^{s} \rightarrow R$ for the $E$-vector optimization problem $\left(\mathrm{VP}_{\mathrm{E}}\right)$ as follows:

$$
\begin{gathered}
L_{E}(x, \lambda, \mu, \xi):=\sum_{i=1}^{p} \lambda_{i}\left(f_{i} \circ E\right)(x)+ \\
\sum_{j=1}^{m} \mu_{j}\left(g_{j} \circ E\right)(x)+\sum_{t=1}^{s} \xi_{t}\left(h_{t} \circ E\right)(x) .
\end{gathered}
$$

Then, we give the definition of a saddle point of the Lagrangian $L_{E}$ defined for $\left(\mathrm{VP}_{\mathrm{E}}\right)$.

\section{Definition 14}

We say that $(\bar{x}, \bar{\lambda}, \bar{\mu}, \bar{\xi}) \in \Omega_{E} \times R_{+}^{p} \times R_{+}^{m} \times R^{s}$ is a saddle point of the $E$-vector optimization problem $\left(V P_{E}\right)$ if:
a) $L_{E}(\bar{x}, \bar{\lambda}, \mu, \xi) \leqq L_{E}(\bar{x}, \bar{\lambda}, \bar{\mu}, \bar{\xi}) \forall \mu \in R_{+}^{m}, \forall \xi \in R^{s}$,
b) $L_{E}(\bar{x}, \bar{\lambda}, \bar{\mu}, \bar{\xi}) \leqq L_{E}(x, \bar{\lambda}, \bar{\mu}, \bar{\xi}) \forall x \in \Omega_{E}$.

We now define a scalar Lagrange function $L: \Omega \times R_{+}^{p} \times R_{+}^{m} \times R^{s} \rightarrow R$ for the considered multiobjective programming problem (VP) as follows:

$$
L(z, \lambda, \mu, \xi):=\sum_{i=1}^{p} \lambda_{i} f_{i}(z)+\sum_{j=1}^{m} \mu_{j} g_{j}(z)+\sum_{t=1}^{s} \xi_{t} h_{t}(z) .
$$

For the scalar Lagrange function $L$ defined above, we now give the definition of its $E$-saddle point.

\section{Definition 15}

Let $\bar{x}$ be a feasible solution of the $E$-vector optimization problem $\left(\mathrm{VP}_{\mathrm{E}}\right)$. We say that $(E(\bar{x}), \bar{\lambda}, \bar{\mu}, \bar{\xi}) \in \Omega \times R_{+}^{p} \times R_{+}^{m} \times R^{s}$ is an $E$-saddle point of the considered multiobjective programming problem (VP) if:

i) $\quad L(E(\bar{x}), \bar{\lambda}, \mu, \xi) \leqq L(E(\bar{x}), \bar{\lambda}, \bar{\mu}, \bar{\xi}) \quad \forall \mu \in R_{+}^{m}, \forall \xi \in R^{s}$,

ii) $L(E(\bar{x}), \bar{\lambda}, \bar{\mu}, \bar{\xi}) \leqq L(z, \bar{\lambda}, \bar{\mu}, \bar{\xi}) \forall z \in \Omega$.

First, we prove the necessary condition for a saddle point of the Lagrangian $L_{E}$ which is defined for the $E$ vector optimization problem $\left(\mathrm{VP}_{\mathrm{E}}\right)$.

\section{Theorem 16}

Let $(\bar{x}, \bar{\lambda}, \bar{\mu}, \bar{\xi}) \in \Omega_{E} \times R_{+}^{p} \times R_{+}^{m} \times R^{s}$ be a saddle point of the scalar Lagrange function $L_{E}$ defined in the problem $\left(\mathrm{VP}_{\mathrm{E}}\right)$. Then, $\bar{x}$ is a weak Pareto solution of $\left(\mathrm{VP}_{\mathrm{E}}\right)$. Further, if the Lagrange multiplier $\bar{\lambda}>0$, then $\bar{x}$ is a Pareto solution of $\left(\mathrm{VP}_{\mathrm{E}}\right)$.

\section{Proof}

By assumption, $(\bar{x}, \bar{\lambda}, \bar{\mu}, \bar{\xi}) \in \Omega_{E} \times R_{+}^{p} \times R_{+}^{m} \times R^{s}$ is a saddle point of the scalar Lagrange function $L_{E}$. Thus, by the condition a) in Definition 14 and (7), the inequality:

$$
\begin{aligned}
& \sum_{i=1}^{p} \bar{\lambda}_{i}\left(f_{i} \circ E\right)(\bar{x})+\sum_{j=1}^{m} \mu_{j}\left(g_{j} \circ E\right)(\bar{x}) \\
& +\sum_{t=1}^{s} \xi_{t}\left(h_{t} \circ E\right)(\bar{x}) \leqq \sum_{I=1}^{p} \bar{\lambda}_{i}\left(f_{i} \circ E\right)(\bar{x}) \\
& +\sum_{j=1}^{m} \bar{\mu}_{j}\left(g_{j} \circ E\right)(\bar{x})+\sum_{t=1}^{s} \bar{\xi}_{t}\left(h_{t} \circ E\right)(\bar{x})
\end{aligned}
$$

holds for all $\mu \in R_{+}^{m}$ and $\xi \in R^{s}$. From the feasibility of $\bar{x}$ in $\left(\mathrm{VP}_{\mathrm{E}}\right)$, we have, for all $\mu \in R_{+}^{m}$ :

$$
\sum_{j=1}^{m} \mu_{j}\left(g_{j} \circ E\right)(\bar{x}) \leqq \sum_{j=1}^{m} \bar{\mu}_{j}\left(g_{j} \circ E\right)(\bar{x})
$$

Then, for $\mu=0,(10)$ gives:

$$
\sum_{j=1}^{m} \bar{\mu}_{j}\left(g_{j} \circ E\right)(\bar{x}) \geqq 0 .
$$

Using the feasibility of $\bar{x}$ in (VPE) together with $\bar{\mu} \in R_{+}^{m}$, we get:

$$
\sum_{j=1}^{m} \bar{\mu}_{j}\left(g_{j} \circ E\right)(\bar{x}) \leqq 0 .
$$


By two inequalities above, it follows that:

$\sum_{J=1}^{m} \bar{\mu}_{j}\left(g_{j} \circ E\right)(\bar{x})=0$

Now, suppose, contrary to the result, that $\bar{x} \in \Omega_{E}$ is not a weak Pareto solution of $\left(\mathrm{VP}_{\mathrm{E}}\right)$. Hence, by Definition 5, there exists $\tilde{x} \in \Omega_{E}$ such that $(f \circ E)(\tilde{x})<(f \circ E)(\bar{x})$. By $\bar{\lambda} \geq 0$, it follows that:

$$
\sum_{i=1}^{p} \bar{\lambda}_{i}\left(f_{i} \circ E\right)(\tilde{x})<\sum_{i=1}^{p} \bar{\lambda}_{i}\left(f_{i} \circ E\right)(\bar{x}) .
$$

Since $\tilde{x} \in \Omega_{E}$ and $\bar{x} \in \Omega_{E},(11)$ and (12) yield:

$$
\begin{aligned}
& \sum_{i=1}^{p} \bar{\lambda}_{i}\left(f_{i} \circ E\right)(\tilde{x})+\sum_{j=1}^{m} \bar{\mu}_{j}\left(g_{j} \circ E\right)(\tilde{x}) \\
& +\sum_{t=1}^{s} \bar{\xi}_{t}(h \circ E)(\tilde{x})<\sum_{i=1}^{p} \bar{\lambda}_{i}\left(f_{i} \circ E\right)(\bar{x}) \\
& +\sum_{j=1}^{m} \bar{\mu}_{j}\left(g_{j} \circ E\right)(\bar{x})+\sum_{t=1}^{s} \bar{\xi}_{t}\left(h_{t} \circ E\right)(\bar{x}) .
\end{aligned}
$$

Hence, by definition of the Lagrange function $L_{E}$ (see (8)), the inequality $L_{E}(\tilde{x}, \bar{\lambda}, \bar{\mu}, \bar{\xi})<L_{E}(\bar{x}, \bar{\lambda}, \bar{\mu}, \bar{\xi})$ is satisfied, which contradicts the inequality $b$ ) in Definition 14.

Now, by using the result established in Theorem 16, we prove the similar result for the original multicriteria optimization problem (VP). Namely, we derive the necessary condition for a saddle point criteria of the Lagrangian $L$ defined for (VP). In other words, we prove the necessary condition for an $E$-saddle point of the scalar Lagrange function $L$ defined in (VP).

\section{Theorem 17}

Let $(E(\bar{x}), \bar{\lambda}, \bar{\mu}, \bar{\xi}) \in \Omega \times R_{+}^{p} \times R_{+}^{m} \times R^{s}$ be an $E$-saddle point of the scalar Lagrangian $L$ defined in the considered vector optimization problem (VP). Then $E(\bar{x})$ is a weak $E$-Pareto solution of (VP). Further, if $\bar{\lambda}>0$, then $E(\bar{x})$ is an $E$-Pareto solution of (VP).

\section{Proof}

We assume that $(E(\bar{x}), \bar{\lambda}, \bar{\mu}, \bar{\xi}) \in \Omega \times R_{+}^{p} \times R_{+}^{m} \times R^{s}$ is an $E$-saddle point of $L$. Then, the conditions (i) and (ii) in Definition 15 are fulfilled. For any $z \in \Omega$, by Lemma 7, there exists $x \in \Omega_{E}$ such that $z=E(x)$. Then, by (8), the conditions (i) and (ii) can be rewritten, respectively:

$$
\begin{gathered}
\sum_{i=1}^{p} \bar{\lambda}_{i}\left(f_{i} \circ E\right)(\bar{x})+\sum_{j=1}^{m} \mu_{j}\left(g_{j} \circ E\right)(\bar{x}) \\
+\sum_{t=1}^{s} \xi_{t}\left(h_{t} \circ E\right)(\bar{x}) \leqq \sum_{i=1}^{p} \bar{\lambda}_{i}\left(f_{i} \circ E\right)(\bar{x}) \\
+\sum_{j=1}^{m} \bar{\mu}_{j}\left(g_{j} \circ E\right)(\bar{x})+\sum_{t=1}^{s} \bar{\xi}_{t}\left(h_{t} \circ E\right)(\bar{x}) \\
\forall \mu \in R_{+}^{m}, \forall \xi \in R^{s}
\end{gathered}
$$

and:

$$
\begin{gathered}
\sum_{i=1}^{p} \bar{\lambda}_{i}\left(f_{i} \circ E\right)(x)+\sum_{j=1}^{m} \bar{\mu}_{j}\left(g_{j} \circ E\right)(x) \\
+\sum_{t=1}^{s} \bar{\xi}_{t}(h \circ E)(x) \leqq \sum_{I=1}^{p} \bar{\lambda}_{i}\left(f_{i} \circ E\right)(\bar{x}) \\
+\sum_{j=1}^{m} \bar{\mu}_{j}\left(g_{j} \circ E\right)(\bar{x})+\sum_{t=1}^{s} \bar{\xi}_{t}\left(h_{t} \circ E\right)(\bar{x}) \forall x \in \Omega_{E} .
\end{gathered}
$$

By (7), inequalities (13) and (14) imply, respectively:

$$
\begin{aligned}
& L_{E}(\bar{x}, \bar{\lambda}, \mu, \xi) \leqq L_{E}(\bar{x}, \bar{\lambda}, \bar{\mu}, \bar{\xi}) \forall \mu \in R_{+}^{m}, \forall \xi \in R^{s}, \\
& L_{E}(\bar{x}, \bar{\lambda}, \bar{\mu}, \bar{\xi}) \leqq L_{E}(x, \bar{\lambda}, \bar{\mu}, \bar{\xi}) \forall x \in \Omega_{E} .
\end{aligned}
$$

Note that (15) and (16) are the saddle point criteria which have already been established in Theorem 16 . Then, by Theorem 16, $\bar{x} \in \Omega_{E}$ is a weak Pareto solution of $\left(\mathrm{VP}_{\mathrm{E}}\right)$. Hence, by Lemma 9 (see also Remark 10), $E(\bar{x})$ is an $E$-Pareto solution of (VP). Thus, the proof of this theorem is completed.

Note that the result proved in Theorem 17 can also be formulated in the following way.

\section{Theorem 18}

Let $\bar{x}$ be a feasible solution of the the Emultiobjective programming problem $\left(\mathrm{VP}_{\mathrm{E}}\right)$ such that $(E(\bar{x}), \bar{\lambda}, \bar{\mu}, \bar{\xi}) \in \Omega \times R_{+}^{p} \times R_{+}^{m} \times R^{s}$ is a saddle point of the scalar Lagrange function $L$ defined for considered multicriteria optimization problem (VP). Then $E(\bar{x})$ is a weak $E$-Pareto solution of the problem (VP). Further, if $\bar{\lambda}>0$, then $E(\bar{x})$ is an $E$-Pareto solution of (VP).

Now, under appropriate $E$-differentiable $E$-convexity hypotheses, we establish the sufficient condition for a saddle point of the Lagrange function $L_{E}$ defined in the $E$-vector optimization problem $\left(\mathrm{VP}_{\mathrm{E}}\right)$ which we use in proving the sufficient condition for an $E$-saddle point of the Lagrange function $L$ defined for the considered multiobjective programming problem (VP).

\section{Theorem 19}

Let $(\bar{x}, \bar{\lambda}, \bar{\mu}, \bar{\xi}) \in \Omega_{E} \times R_{+}^{p} \times R_{+}^{m} \times R^{s}$ be a Karush-KuhnTucker point of the $E$-vector optimization problem $\left(\mathrm{VP}_{\mathrm{E}}\right)$. Assume, moreover, that each objective function $f_{i}$, $i \in I$, is $E$-convex at $\bar{x}$ on $\Omega_{E}$, each constraint function $g_{j}, j \in J, \quad$ is $E$-convex at $\bar{x}$ on $\Omega_{E}, h_{t}$, $t \in T_{E}^{+}(\bar{x})=\left\{t \in T: \bar{\xi}_{t}>0\right\}$ and the functions $-h_{t}$, $t \in T_{E}^{-}(\bar{x})=\left\{t \in T: \bar{\xi}_{t}<0\right\}$, are $E$-convex at $\bar{x}$ on $\Omega_{E}$. Then $(\bar{x}, \bar{\lambda}, \bar{\mu}, \bar{\xi})$ is a saddle point of the Lagrange function $L_{E}$ defined in the problem $\left(\mathrm{VP}_{\mathrm{E}}\right)$. 


\section{Proof}

First, we prove the inequality (i) in Definition 14. By hypothesis $(\bar{x}, \bar{\lambda}, \bar{\mu}, \bar{\xi}) \in \Omega_{E} \times R_{+}^{p} \times R_{+}^{m} \times R^{s}$ is a KKT point of the $E$-vector optimization problem $\left(\mathrm{VP}_{\mathrm{E}}\right)$. Then, by $\bar{x} \in \Omega_{E}$, we have that the relations:

$$
\xi_{t}\left(h_{t} \circ E\right)(\bar{x})=\bar{\xi}_{t}\left(h_{t} \circ E\right)(\bar{x}), t \in T
$$

hold for all $\xi=\left(\xi_{1}, \ldots, \xi_{s}\right) \in R^{s}$. Again by $\bar{x} \in \Omega_{E}$ and using the $E$-KKT necessary optimality condition (5), we get that the inequalities:

$$
\mu_{j}\left(g_{j} \circ E\right)(\bar{x}) \leqq \bar{\mu}_{j}\left(g_{j} \circ E\right)(\bar{x}), j \in J
$$

hold for all $\mu=\left(\mu_{1}, \ldots, \mu_{m}\right) \in R_{+}^{m}$. By (17) and (18), we have, for all $\mu \in R_{+}^{m}$ and for all $\xi \in R^{s}$ :

$$
\begin{aligned}
& \sum_{i=1}^{p} \bar{\lambda}_{i}\left(f_{i} \circ E\right)(\bar{x})+\sum_{j=1}^{m} \mu_{j}\left(g_{j} \circ E\right)(\bar{x}) \\
& +\sum_{t=1}^{s} \xi_{t}\left(h_{t} \circ E\right)(\bar{x}) \leqq \sum_{i=1}^{p} \bar{\lambda}_{i}\left(f_{i} \circ E\right)(\bar{x}) \\
& +\sum_{j=1}^{m} \bar{\mu}_{j}\left(g_{j} \circ E\right)(\bar{x})+\sum_{t=1}^{s} \bar{\xi}_{t}\left(h_{t} \circ E\right)(\bar{x}) .
\end{aligned}
$$

Then, by (8), the inequality:

$$
L_{E}(\bar{x}, \bar{\lambda}, \mu, \xi) \leqq L_{E}(\bar{x}, \bar{\lambda}, \bar{\mu}, \bar{\xi})
$$

holds for all $\mu \in R_{+}^{m}$ and $\xi \in R^{s}$. Hence, the inequality i) in Definition 14 is fulfilled.

We now prove the condition (ii) in Definition 14. From the assumption, $f_{i}, i \in I, g_{j}, j \in J, h_{t}, t \in T_{E}^{+}(\bar{x}),-h_{t}$, $t \in T_{E}^{-}(\bar{x})$, are $E$-convex at $\bar{x}$ on $\Omega_{E}$. Then, by Proposition 4 and the KKT optimality condition (6), the inequalities:

$$
\begin{gathered}
\bar{\lambda}_{i} f_{i}(E(x))-\bar{\lambda}_{i} f_{i}(E(\bar{x})) \geqq \\
\bar{\lambda}_{i} \nabla f_{i}(E(\bar{x}))(E(x)-E(\bar{x})), i \in I, \\
\bar{\mu}_{j} g_{j}(E(x))-\bar{\mu}_{j} g_{j}(E(\bar{x})) \geqq \\
\bar{\mu}_{j} \nabla g_{j}(E(\bar{x}))(E(x)-E(\bar{x})), j \in J(E(\bar{x})), \\
\bar{\xi}_{t} h_{t}(E(x))-\bar{\xi}_{t} h_{t}(E(\bar{x})) \geqq \\
\bar{\xi}_{t} \nabla h_{t}(E(\bar{x}))(E(x)-E(\bar{x})), t \in T^{+}(E(\bar{x})), \\
\bar{\xi}_{t} h_{t}(E(x))-\bar{\xi}_{t} h_{t}(E(\bar{x})) \geqq \\
\bar{\xi}_{t} \nabla h_{t}(E(\bar{x}))(E(x)-E(\bar{x})), t \in T^{-}(E(\bar{x}))
\end{gathered}
$$

hold for all $x \in \Omega_{E}$. Hence, we add both sides of each inequality (20)-(23). Thus, by the KKT necessary optimality condition (4), we get that the inequality:

$$
\begin{aligned}
& \sum_{i=1}^{p} \bar{\lambda}_{i}\left(f_{i} \circ E\right)(x)+\sum_{j=1}^{m} \bar{\mu}_{j}\left(g_{j} \circ E\right)(x) \\
& +\sum_{t=1}^{s} \bar{\xi}_{t}\left(h_{t} \circ E\right)(x) \geqq \sum_{I=1}^{p} \bar{\lambda}_{i}\left(f_{i} \circ E\right)(\bar{x}) \\
& +\sum_{j=1}^{m} \bar{\mu}_{j}\left(g_{j} \circ E\right)(\bar{x})+\sum_{t=1}^{s} \bar{\xi}_{t}\left(h_{t} \circ E\right)(\bar{x})
\end{aligned}
$$

is satisfied for all $x \in \Omega_{E}$. Then, by (7), the inequality:

$$
L_{E}(x, \bar{\lambda}, \bar{\mu}, \bar{\xi}) \geqq L_{E}(\bar{x}, \bar{\lambda}, \bar{\mu}, \bar{\xi})
$$

holds for all $x \in \Omega_{E}$. Hence, by (19) and (24), $(\bar{x}, \bar{\lambda}, \bar{\mu}, \bar{\xi})$ is a saddle point of the scalar Lagrangian $L_{E}$. Thus, the conclusion of this theorem is established.

\section{Corollary 20}

Let $\bar{x} \in \Omega_{E}$ be a (weak) Pareto solution of the $E$ vector optimization problem $\left(\mathrm{VP}_{\mathrm{E}}\right)$ and all assumptions of Theorem 19 be satisfied. Then, there exist Lagrange multipliers $\bar{\lambda} \in R^{p}, \quad \bar{\mu} \in R^{m}$ and $\bar{\xi} \in R^{s}$ such that $(\bar{x}, \bar{\lambda}, \bar{\mu}, \bar{\xi})$ is a saddle point of the Lagrangian $L_{E}$ defined for $\left(\mathrm{VP}_{\mathrm{E}}\right)$.

By using Theorem 19 and Corollary 20, we now prove the sufficient condition for an $E$-saddle point of the Lagrange function $\mathrm{L}$ in the problem (VP).

\section{Theorem 21}

Let $\bar{x}$ be a feasible point of the $E$-vector optimization problem $\left(\mathrm{VP}_{\mathrm{E}}\right)$ such that $(E(\bar{x}), \bar{\lambda}, \bar{\mu}, \bar{\xi}) \in \Omega \times R_{+}^{p} \times R_{+}^{m} \times R^{s}$ is an $E$-KKT point of the considered multiobjective programming problem (VP). Assume, moreover, that each objective function $f_{i}, i \in I$, is $E$-convex at $\bar{x}$ on $\Omega_{E}$, each constraint function $g_{j}, j \in J$, is $E$-convex at $\bar{x}$ on $\Omega_{E}$, each constraint function $h_{t}, t \in T_{E}^{+}(\bar{x})$ and each function $-h_{t}, t \in T_{E}^{-}(\overline{\mathrm{x}})$, are $E$-convex at $\bar{x}$ on $\Omega_{E}$. Then $(E(\bar{x}), \bar{\lambda}, \bar{\mu}, \bar{\xi}) \in \Omega \times R_{+}^{p} \times R_{+}^{m} \times R^{s}$ is an $E$-saddle point of the Lagrangian $L$ defined for (VP).

\section{Proof}

By assumption, $\bar{x}$ is a feasible solution of the $E$ vector optimization problem $\left(\mathrm{VP}_{\mathrm{E}}\right)$ such that $(E(\bar{x}), \bar{\lambda}, \bar{\mu}, \bar{\xi}) \in \Omega \times R_{+}^{p} \times R_{+}^{m} \times R^{s}$ is an $E$-KKT point of the considered multiobjective programming problem (VP). Then, by Definition 13, the E-KKT conditions are fulfilled for the problem (VP) with Lagrange multipliers $\bar{\lambda}, \bar{\mu}, \bar{\xi}$. Since all hypotheses of Theorem 19 are fulfilled, $(\bar{x}, \bar{\lambda}, \bar{\mu}, \bar{\xi}) \in \Omega_{E} \times R_{+}^{p} \times R_{+}^{m} \times R^{s}$ is a saddle point of the Lagrange function $L_{E}$ in $\left(\mathrm{VP}_{\mathrm{E}}\right)$. This means, by Definition 14, that the following conditions: 


$$
\begin{aligned}
& L_{E}(\bar{x}, \bar{\lambda}, \mu, \bar{\xi}) \leqq L_{E}(\bar{x}, \bar{\lambda}, \bar{\mu}, \bar{\xi}) \forall \mu \in R_{+}^{m}, \forall \xi \in R^{s}, \\
& L_{E}(\bar{x}, \bar{\lambda}, \bar{\mu}, \bar{\xi}) \leqq L_{E}(x, \bar{\lambda}, \bar{\mu}, \bar{\xi}) \forall x \in \Omega_{E}
\end{aligned}
$$

hold. By (7), it follows that the inequalities:

$$
\begin{gathered}
\sum_{i=1}^{p} \bar{\lambda}_{i}\left(f_{i} \circ E\right)(\bar{x})+\sum_{j=1}^{m} \mu_{j}\left(g_{j} \circ E\right)(\bar{x}) \\
+\sum_{t=1}^{s} \xi_{t}\left(h_{t} \circ E\right)(\bar{x}) \leqq \sum_{i=1}^{p} \bar{\lambda}_{i}\left(f_{i} \circ E\right)(\bar{x}) \\
+\sum_{j=1}^{m} \bar{\mu}_{j}\left(g_{j} \circ E\right)(\bar{x})+\sum_{t=1}^{s} \bar{\xi}_{t}\left(h_{t} \circ E\right)(\bar{x}) \\
\forall \mu \in R_{+}^{m}, \forall \xi \in R^{s} \\
\sum_{i=1}^{p} \bar{\lambda}_{i}\left(f_{i} \circ E\right)(x)+\sum_{j=1}^{m} \bar{\mu}_{j}\left(g_{j} \circ E\right)(x) \\
+\sum_{t=1}^{s} \bar{\xi}_{t}\left(h_{t} \circ E\right)(x) \geqq \sum_{i=1}^{p} \bar{\lambda}_{i}\left(f_{i} \circ E\right)(\bar{x}) \\
+\sum_{j=1}^{m} \bar{\mu}_{j}\left(g_{j} \circ E\right)(\bar{x})+\sum_{t=1}^{s} \bar{\xi}_{t}\left(h_{t} \circ E\right)(\bar{x}) \quad \forall x \in \Omega_{E}
\end{gathered}
$$

hold, respectively. Since $E: R^{n} \rightarrow R^{n}$ is an one-to-one and onto operator, this means that, for any $x \in \Omega_{E}$, there existas $z \in \Omega$, such that $z=E(x)$. Hence, (25) and (26) yield, respectively:

$$
\begin{aligned}
& \sum_{i=1}^{p} \bar{\lambda}_{i}\left(f_{i} \circ E\right)(\bar{x})+\sum_{j=1}^{m} \mu_{j}\left(g_{j} \circ E\right)(\bar{x}) \\
& +\sum_{t=1}^{s} \xi_{t}\left(h_{t} \circ E\right)(\bar{x}) \leqq \sum_{I=1}^{p} \bar{\lambda}_{i}\left(f_{i} \circ E\right)(\bar{x}) \\
& +\sum_{j=1}^{m} \bar{\mu}_{j}\left(g_{j} \circ E\right)(\bar{x})+\sum_{t=1}^{s} \bar{\xi}_{t}\left(h_{t} \circ E\right)(\bar{x}) \\
& \forall \mu \in R_{+}^{m}, \forall \xi \in R^{s} \\
& \sum_{i=1}^{p} \bar{\lambda}_{i} f_{i}(z)+\sum_{j=1}^{m} \bar{\mu}_{j} g_{j}(z)+\sum_{t=1}^{s} \bar{\xi}_{t} h_{t}(z) \\
& \geqq \sum_{I=1}^{p} \bar{\lambda}_{i}\left(f_{i} \circ E\right)(\bar{x})+\sum_{j=1}^{m} \bar{\mu}_{j}\left(g_{j} \circ E\right)(\bar{x}) \\
& +\sum_{t=1}^{s} \bar{\xi}_{t}\left(h_{t} \circ E\right)(\bar{x}) \forall x \in \Omega .
\end{aligned}
$$

By Definition 15, (27) and (28) imply that $(E(\bar{x}), \bar{\lambda}, \bar{\mu}, \bar{\xi}) \in \Omega \times R_{+}^{p} \times R_{+}^{m} \times R^{s}$ is an $E$-saddle point of the Lagrange function $L$ defined for (VP).

As it follows from the above proof, the definition of an $E$-saddle point of the Lagrange function $L$ can be reformulated as follows.

\section{Definition 22}

Let $\bar{x}$ be a feasible solution of the $E$-vector optimization problem (VPE). We say that $(E(\bar{x}), \bar{\lambda}, \bar{\mu}, \bar{\xi}) \in \Omega \times R_{+}^{p} \times R_{+}^{m} \times R^{s}$ is an $E$-saddle point for the considered multiobjective programming problem (VP) if:

i. $\quad L(E(\bar{x}), \bar{\lambda}, \mu, \xi) \leqq L(E(\bar{x}), \bar{\lambda}, \bar{\mu}, \bar{\xi}) \forall \mu \in R_{+}^{m}, \forall \xi \in R^{s}$,

ii. $\quad L(E(\bar{x}), \bar{\lambda}, \bar{\mu}, \bar{\xi}) \leqq L(E(x), \bar{\lambda}, \bar{\mu}, \bar{\xi}) \forall E(x) \in \Omega$.
Now, we illustrate the main result established in this section by an example of an $E$-differentiable multiobjective programming problem.

\section{Example 23}

Consider the following nonconvex nondifferentiable vector optimization problem:

$$
\begin{aligned}
\text { minimize } f\left(x_{1}, x_{2}\right) & =\left(x_{1}^{2}+2 x_{1}-\sqrt[3]{x_{2}}, \sqrt[3]{x_{2}}+x_{2}-x_{1}\right) \\
\text { s.t. } g\left(x_{1}, x_{2}\right) & =\sqrt[3]{x_{2}}-x_{1} \leqq 0,(V P 1) \\
h\left(x_{1}, x_{2}\right) & =x_{1}^{2}-\sqrt[3]{x_{2}}=0 .
\end{aligned}
$$

Note that:

$$
\Omega=\left\{\left(x_{1}, x_{2}\right) \in R^{2}: \sqrt[3]{x_{2}}-x_{1} \leqq 0 \wedge x_{1}^{2}-\sqrt[3]{x_{2}}=0\right\} .
$$

Let $E: R^{2} \rightarrow R^{2}$ be an one-to-one and onto mapping defined by $E\left(x_{1}, x_{2}\right)=\left(x_{1}, x_{2}^{3}\right)$. Now, for the considered nonconvex nondifferentiable multicriteria optimization problem (VP1), we construct its associated $E$-vector optimization problem $\left(\mathrm{VP} 1_{\mathrm{E}}\right)$ as follows:

$$
\begin{aligned}
\text { minimize } f\left(E\left(x_{1}, x_{2}\right)\right) & =\left(x_{1}^{2}+2 x_{1}-x_{2}, x_{2}^{3}+x_{2}-x_{1}\right) \\
\text { s.t. } g\left(E\left(x_{1}, x_{2}\right)\right) & =x_{2}-x_{1} \leqq 0, \quad\left(V P 1_{E}\right) \\
h\left(E\left(x_{1}, x_{2}\right)\right) & =x_{1}^{2}-x_{2}=0 .
\end{aligned}
$$

Note that $\Omega_{E}=\left\{\left(x_{1}, x_{2}\right) \in R^{2}: x_{2}-x_{1} \leqq 0 \wedge x_{1}^{2}-x_{2}=0\right\}$. and $\bar{x}=(0,0)$ is a feasible solution of the problem $\left(\mathrm{VP} 1_{\mathrm{E}}\right)$. Further, note that all functions constituting the considered vector optimization problem (VP1) are $E$ differentiable at $\bar{x}=(0,0)$. Moreover, the E-KKT necessary optimality conditions (4)-(6) are satisfied at $\bar{x}=(0,0)$ with Lagrange multipliers $\bar{\lambda}_{1}=\frac{1}{2}, \bar{\lambda}_{2}=\frac{1}{4}, \bar{\mu}=\frac{3}{4}$ and $\bar{\xi}=\frac{1}{2}$. Hence, we have shown that all hypotheses of Theorem 19 are satisfied. Thus, we conclude by Theorem 19 that $(\bar{x}, \bar{\lambda}, \bar{\mu}, \bar{\xi})$ is a saddle point of the Lagrange function $L_{E}$ defined for the $E$-vector optimization problem $\left(\mathrm{VP} 1_{\mathrm{E}}\right)$. Further, it follows by Theorem 21 that $(E(\bar{x}) \bar{\lambda}, \bar{\mu}, \bar{\xi})$ is an $E$-saddle point of the Lagrange function $L$ defined for the considered multiobjective programming problem (VP1).

\section{Vector $\boldsymbol{E}$-saddle Point Criteria}

the

In this section, we introduce a definition of vector-valued Lagrange function 
$L_{p_{E}}: \Omega_{E} \times R^{p} \times R^{m} \times R^{s} \rightarrow R^{p}$ defined for the constrained $E$-vector optimization problem $\left(\mathrm{VP}_{\mathrm{E}}\right)$. Namely, this Lagrange function is defined as follows:

$L_{p_{E}}(x, \lambda, \mu, \xi):=\operatorname{diag} \lambda(f \circ E)(x)+$

$\frac{1}{p}\left[\sum_{j=1}^{m} \mu_{j}\left(g_{j} \circ E\right)(x)+\sum_{t=1}^{s} \xi_{t}\left(h_{t} \circ E\right)(x)\right] e$,

where, $e=[1, \ldots, 1] \in R^{p}$ and, moreover,

$$
\operatorname{diag} \lambda=\left[\begin{array}{cccc}
\lambda_{1} & 0 & \cdots & 0 \\
0 & \lambda_{2} & \cdots & 0 \\
\vdots & \vdots & \ddots & \vdots \\
0 & 0 & \cdots & \lambda_{p}
\end{array}\right]
$$

\section{Definition 24}

We say that $(\bar{x}, \bar{\lambda}, \bar{\mu}, \bar{\xi}) \in \Omega_{E} \times R_{+}^{p} \times R_{+}^{m} \times R^{s}$ is a saddle point of the vector-valued Lagrange function $L_{p_{E}}$ defined in the $E$-vector optimization problem $\left(\mathrm{VP}_{\mathrm{E}}\right)$ if:

a) $L_{p_{E}}(\bar{x}, \bar{\lambda}, \mu, \xi) \leqq L_{p_{E}}(\bar{x}, \bar{\lambda}, \bar{\mu}, \bar{\xi}) \forall \mu \in R_{+}^{m}, \forall \xi \in R^{s}$,

b) $L_{p_{E}}(x, \bar{\lambda}, \bar{\mu}, \bar{\xi}) \notin L_{p_{E}}(\bar{x}, \bar{\lambda}, \bar{\mu}, \bar{\xi}) \forall x \in \Omega_{E}$.

Now, for the considered multiobjective programming problem (VP), we define its vector-valued Lagrange function $L_{p}: \Omega \times R_{+}^{p} \times R_{+}^{m} \times R^{s} \rightarrow R$ by:

$$
\begin{gathered}
L_{p}(z, \lambda, \mu, \xi):=\operatorname{diag} \lambda f(z)+ \\
\frac{1}{p}\left[\sum_{j=1}^{m} \mu_{j} g_{j}(z)+\sum_{t=1}^{s} \xi_{t} h_{t}(z)\right] e .
\end{gathered}
$$

\section{Definition 25}

Let $\bar{x}$ be a feasible solution of the $E$-vector optimization problem $\left(\mathrm{VP}_{\mathrm{E}}\right)$. We say that $(E(\bar{x}), \bar{\lambda}, \bar{\mu}, \bar{\xi}) \in \Omega \times R_{+}^{p} \times R_{+}^{m} \times R^{s}$ is a saddle point of the considered multiobjective programming problem (VP) if:

i. $\quad L_{p}(E(\bar{x}), \bar{\lambda}, \mu, \bar{\xi}) \leqq L_{p}(E(\bar{x}), \bar{\lambda}, \bar{\mu}, \bar{\xi}) \forall \mu \in R_{+}^{m}, \forall \xi \in R^{s}$,

ii. $\quad L_{p}(z, \bar{\lambda}, \bar{\mu}, \bar{\xi}) \not L_{p}(E(\bar{x}), \bar{\lambda}, \bar{\mu}, \bar{\xi}) \forall z \in \Omega$.

First, we prove the necessary condition for a vector saddle point of the Lagrange function $L_{p_{E}}$.

\section{Theorem 26}

Let $(\bar{x}, \bar{\lambda}, \bar{\mu}, \bar{\xi}) \in \Omega_{E} \times R_{+}^{p} \times R_{+}^{m} \times R^{s}$ be a saddle point of the vector-valued Lagrange function $L_{p_{E}}$ defined for the
$E$-vector optimization problem $\left(\mathrm{VP}_{\mathrm{E}}\right)$. Then $\bar{x}$ is a weak Pareto solution of $\left(\mathrm{VP}_{\mathrm{E}}\right)$. If Lagrange multiplier $\bar{\lambda}$ is extra assumed to satisfy $\bar{\lambda}>0$, then $\bar{x}$ is a Pareto solution of $\left(\mathrm{VP}_{\mathrm{E}}\right)$.

\section{Proof}

Since $(\bar{x}, \bar{\lambda}, \bar{\mu}, \bar{\xi}) \in \Omega_{E} \times R_{+}^{p} \times R_{+}^{m} \times R^{s}$ is a saddle point of the vector-valued Lagrange function $L_{p_{E}}$, by Definition 24, the conditions a) and b) are satisfied. Thus, by the condition a) in Definition 24 and (29), the inequality:

$$
\begin{gathered}
\bar{\lambda}_{i}\left(f_{i} \circ E\right)(\bar{x})+\frac{1}{p}\left[\sum_{j=1}^{m} \mu_{j}\left(g_{j} \circ E\right)(\bar{x})\right. \\
\left.+\sum_{t=1}^{s} \xi_{t}\left(h_{t} \circ E\right)(\bar{x})\right] \leqq \bar{\lambda}_{i}\left(f_{i} \circ E\right)(\bar{x}) \\
+\frac{1}{p}\left[\sum_{j=1}^{m} \bar{\mu}_{j}\left(g_{j} \circ E\right)(\bar{x})+\sum_{t=1}^{s} \bar{\xi}_{t}\left(h_{t} \circ E\right)(\bar{x})\right] i=1, \ldots, p
\end{gathered}
$$

holds for all $\mu \in R_{+}^{m}$ and for all $\xi \in R^{s}$. From the feasibility of $\bar{x}$ in the problem $\left(\mathrm{VP}_{\mathrm{E}}\right)$, it follows that the inequality:

$$
\sum_{j=1}^{m} \mu_{j}\left(g_{j} \circ E\right)(\bar{x}) \leqq \sum_{j=1}^{m} \bar{\mu}_{j}\left(g_{j} \circ E\right)(\bar{x})
$$

holds for all $\mu \in R_{+}^{m}$. In the similar way as in the proof of Theorem 16, we get:

$$
\sum_{j=1}^{m} \bar{\mu}_{j}\left(g_{j} \circ E\right)(\bar{x})=0 .
$$

Now, suppose, contrary to the result, that $\bar{x} \in \Omega_{E}$ is not a Pareto solution of the problem $\left(\mathrm{VP}_{\mathrm{E}}\right)$. Then, there exists $\tilde{x} \in \Omega_{E}$ such that:

$$
(f \circ E)(\tilde{x}) \leq(f \circ E)(\bar{x}) .
$$

By assumption, the Lagrange multiplier $\bar{\lambda}$ is assumed to satisfy $\bar{\lambda}>0$. Thus:

$$
\operatorname{diag} \bar{\lambda}(f \circ E)(\tilde{x}) \leq \operatorname{diag} \bar{\lambda}(f \circ E)(\bar{x}) .
$$

Using the feasibility of $\tilde{x}$ and $\bar{x}$ of $\left(\mathrm{VP}_{\mathrm{E}}\right)$ together with (31), we get:

$$
\begin{gathered}
\operatorname{diag} \bar{\lambda}(f \circ E)(\tilde{x})+\frac{1}{p}\left[\sum_{j=1}^{m} \bar{\mu}_{j}\left(g_{j} \circ E\right)(\tilde{x})\right. \\
\left.+\sum_{t=1}^{s} \bar{\xi}_{t}\left(h_{t} \circ E\right)(\tilde{x})\right] e \leq \operatorname{diag} \bar{\lambda}(f \circ E)(\bar{x}) \\
+\frac{1}{p}\left[\sum_{j=1}^{m} \bar{\mu}_{j}\left(g_{j} \circ E\right)(\bar{x})+\sum_{t=1}^{s} \bar{\xi}_{t}\left(h_{t} \circ E\right)(\bar{x})\right] e .
\end{gathered}
$$

Hence, by (29), (34) implies that the inequality: 


$$
L_{p_{E}}(\tilde{x}, \bar{\lambda}, \bar{\mu}, \bar{\xi}) \leq L_{p_{E}}(\bar{x}, \bar{\lambda}, \bar{\mu}, \bar{\xi})
$$

holds, which is a contradiction to the condition ii) in Definition 24.

\section{Remark 27}

Note that it is sufficient to assume in Theorem 26 that $\bar{\lambda} \geq 0$ in order to prove that $\bar{x}$ is a weak Pareto optimal of $\left(\mathrm{VP}_{\mathrm{E}}\right)$.

Using the result established in Theorem 28, we prove the necessary condition for $(E(\bar{x}), \bar{\lambda}, \bar{\mu}, \bar{\xi}) \in \Omega \times R_{+}^{p} \times R_{+}^{m} \times R^{s}$ to be a vector $E$-saddle point of the Lagrange function $L_{p}$ in (VP).

\section{Theorem 28}

Let $\bar{x}$ be a feasible solution of the $E$-vector optimization problem $\left(\mathrm{VP}_{\mathrm{E}}\right)$ such that $(E(\bar{x}), \bar{\lambda}, \bar{\mu}, \bar{\xi}) \in \Omega \times R_{+}^{p} \times R_{+}^{m} \times R^{s}$ is an $E$-saddle point of the vector-valued Lagrange function $L_{p}$ defined for the considered multiobjective programming problem (VP). Then $E(\bar{x})$ is a weak $E$-Pareto solution of (VP). If we assume extra that Lagrange multiplier $\bar{\lambda}>0$, then $E(\bar{x})$ is an E-Pareto solution in (VP).

\section{Proof}

Assume that $\bar{x}$ is a feasible solution of the $E$-vector optimization problem $\left(\mathrm{VP}_{\mathrm{E}}\right)$ such that $(E(\bar{x}), \bar{\lambda}, \bar{\mu}, \bar{\xi}) \in \Omega \times R_{+}^{p} \times R_{+}^{m} \times R^{s}$ is an $E$-saddle point of the vector-valued Lagrange function $L_{p}$. Then, by Definition 25, the conditions (i) and (ii) are fulfilled. For any $z \in \Omega$, by Lemma 7 , there exists $x \in \Omega_{E}$ such that $z=$ $E(x)$. Then, by (30), the following relations:

$$
\begin{gathered}
\operatorname{diag} \bar{\lambda}(f \circ E)(\bar{x})+\frac{1}{p}\left[\sum_{j=1}^{m} \mu_{j}\left(g_{j} \circ E\right)(\bar{x})\right. \\
\left.+\sum_{t=1}^{s} \xi_{t}\left(h_{t} \circ E\right)(\bar{x})\right] e \leqq \operatorname{diag} \bar{\lambda}(f \circ E)(\bar{x}) \\
+\frac{1}{p}\left[\sum_{j=1}^{m} \bar{\mu}_{j}\left(g_{j} \circ E\right)(\bar{x})+\sum_{t=1}^{s} \bar{\xi}_{t}\left(h_{t} \circ E\right)(\bar{x})\right] e, i=1, \ldots, p
\end{gathered}
$$

and:

$$
\begin{gathered}
\operatorname{diag} \bar{\lambda}(f \circ E)(x)+\frac{1}{p}\left[\sum_{j=1}^{m} \bar{\mu}_{j}\left(g_{j} \circ E\right)(x)\right. \\
\left.+\sum_{t=1}^{s} \bar{\xi}_{t}\left(h_{t} \circ E\right)(x)\right] e \not \operatorname{diag} \bar{\lambda}(f \circ E)(\bar{x}) \\
+\frac{1}{p}\left[\sum_{j=1}^{m} \bar{\mu}_{j}\left(g_{j} \circ E\right)(\bar{x})+\sum_{t=1}^{s} \bar{\xi}_{t}\left(h_{t} \circ E\right)(\bar{x})\right] e
\end{gathered}
$$

hold for all $\mu \in R_{+}^{m}, \xi \in R^{s}$ and any $E(x) \in \Omega$. By (35), (36) and (29), we conclude that the conditions (a) and (b) in Definition 24 are satisfied. Since all hypotheses of Theorem 26 are fulfilled, $\bar{x} \in \Omega$ is a (weak) Pareto solution of $\left(\mathrm{VP}_{\mathrm{E}}\right)$. Hence, by Lemma 9 (see also Remark $10), E(\bar{x})$ is a (weak) E-Pareto solution of (VP). This completes the proof of this theorem.

Now, we prove the sufficient condition for $(\bar{x}, \bar{\lambda}, \bar{\mu}, \bar{\xi}) \in \Omega_{E} \times R^{p} \times R^{m} \times R^{s}$ to be a vector saddle point of the Lagrange function $L_{p_{E}}$.

\section{Theorem 29}

Let $(\bar{x}, \bar{\lambda}, \bar{\mu}, \bar{\xi}) \in \Omega_{E} \times R^{p} \times R^{m} \times R^{s}$ be an Karush-KuhnTucker point of the vector-valued Lagrange function $L_{p_{E}}$. Assume, moreover, that each objective function $f_{i}, i \in I$, is $E$-convex at $\bar{x}$ on $\Omega_{E}$, each constraint function $g_{j}, j \in J$, is $E$-convex at $\bar{x}$ on $\Omega_{E}$, the functions $h_{t}, t \in T_{E}^{+}(\bar{x})$ and the functions $-h_{t}, t \in T_{E}^{-}(\bar{x})$, are $E$-convex at $\bar{x}$ on $\Omega_{E}$. Then $(\bar{x}, \bar{\lambda}, \bar{\mu}, \bar{\xi})$ is a saddle point of $L_{p_{E}}$.

\section{Proof}

First, we prove the inequality (a) in Definition 24. By assumption, $(\bar{x}, \bar{\lambda}, \bar{\mu}, \bar{\xi}) \in \Omega_{E} \times R^{p} \times R^{m} \times R^{s}$ is a KarushKuhn-Tucker point of $L_{p_{E}}$. Hence, from the feasibility of $\bar{x}$, we have that the inequalities:

$\xi_{t} \nabla\left(h_{t} \circ E\right)(\bar{x})=\bar{\xi}_{t} \nabla\left(h_{t} \circ E\right)(\bar{x}), t \in T$

hold for all $\xi=\left(\xi_{1}, \ldots, \xi_{s}\right) \in R^{s}$. By $\bar{x} \in \Omega_{E}$ and the $E$-KKT necessary optimality condition (5), it follows that the inequalities:

$\mu_{j} \nabla\left(g_{j} \circ E\right)(\bar{x}) \leqq \bar{\mu}_{j} \nabla\left(g_{j} \circ E\right)(\bar{x}), j \in J$

are satisfied for all $\mu=\left(\mu_{1}, \ldots, \mu_{m}\right) \in R_{+}^{m}$. Combining (37) and (38), we get, for all $\mu \in R_{+}^{m}$ and $\xi \in R^{s}$ :

$$
\begin{gathered}
\operatorname{diag} \bar{\lambda}(f \circ E)(\bar{x})+\frac{1}{p}\left[\sum_{j=1}^{m} \mu_{j}\left(g_{j} \circ E\right)(\bar{x})\right. \\
\left.+\sum_{t=1}^{s} \xi_{t}\left(h_{t} \circ E\right)(\bar{x})\right] e \leq \operatorname{diag} \bar{\lambda}(f \circ E)(\bar{x}) \\
+\frac{1}{p}\left[\sum_{j=1}^{m} \bar{\mu}_{j}\left(g_{j} \circ E\right)(\bar{x})+\sum_{t=1}^{s} \bar{\xi}_{t}\left(h_{t} \circ E\right)(\bar{x})\right] e .
\end{gathered}
$$

Then, by (29), the inequality:

$$
L_{p_{E}}(\bar{x}, \bar{\lambda}, \mu, \xi) \leqq L_{p_{E}}(\bar{x}, \bar{\lambda}, \bar{\mu}, \bar{\xi})
$$

holds for all $\mu \in R_{+}^{m}$ and $\xi \in R^{s}$. 
We now establish the condition (b) in Definition 24. We prove it by means of contradiction. Suppose that there exists $\tilde{x} \in \Omega_{E}$ such that

$$
L_{p_{E}}(\tilde{x}, \bar{\lambda}, \bar{\mu}, \bar{\xi}) \leq L_{p_{\bar{E}}}(\bar{x}, \bar{\lambda}, \bar{\mu}, \bar{\xi}) .
$$

Then, by (29), we have:

$$
\begin{aligned}
& \sum_{i=1}^{k} \bar{\lambda}_{i}\left(f_{i} \circ E\right)(\tilde{x})+\sum_{j=1}^{m} \bar{\mu}_{j}\left(g_{j} \circ E\right)(\tilde{x}) \\
& +\sum_{t=1}^{s} \bar{\xi}_{t}\left(h_{t} \circ E\right)(\tilde{x})<\sum_{i=1}^{k} \bar{\lambda}_{i}\left(f_{i} \circ E\right)(\bar{x}) \\
& +\sum_{j=1}^{m} \bar{\mu}_{j}\left(g_{j} \circ E\right)(\bar{x})+\sum_{t=1}^{s} \bar{\xi}_{t}\left(h_{t} \circ E\right)(\bar{x}) .
\end{aligned}
$$

Since $f_{i}, i \in I, g_{j}, j \in J, h_{t}, t \in T_{E}^{+}(\bar{x}),-h_{t}, t \in T_{E}^{-}(\bar{x})$, are $E$-convex on $\Omega_{E}$, by Proposition 4 , the inequalities:

$$
\begin{gathered}
f_{i}(E(\tilde{x}))-f_{i}(E(\bar{x})) \geqq \nabla f_{i}(E(\bar{x}))(E(\tilde{x})-E(\bar{x})), i \in I, \\
g_{j}(E(\tilde{x}))-g_{j}(E(\bar{x})) \geqq \\
\nabla g_{j}(E(\bar{x}))(E(\tilde{x})-E(\bar{x})), j \in J(E(\bar{x})), \\
h_{t}(E(\tilde{x}))-h_{t}(E(\bar{x})) \geqq \\
\nabla h_{t}(E(\bar{x}))(E(\tilde{x})-E(\bar{x})), t \in T^{+}(E(\bar{x})), \\
-h_{t}(E(\tilde{x}))+h_{t}(E(\bar{x})) \geqq \\
-\nabla h_{t}(E(\bar{x}))(E(\tilde{x})-E(\bar{x})), t \in T^{-}(E(\bar{x}))
\end{gathered}
$$

hold, respectively. Then, we multiply inequalities (41)-(44) by corresponding Lagrange multipliers, respectively. After that, we add the resulting inequalities and obtain:

$$
\begin{aligned}
& \sum_{i=1}^{k} \bar{\lambda}_{i}\left(f_{i} \circ E\right)(\tilde{x})+\sum_{j \in J(E(\tilde{)}))} \bar{\mu}_{j}\left(g_{j} \circ E\right)(\tilde{x}) \\
& +\sum_{t \in T_{E}^{+}(\bar{x})}^{s} \bar{\xi}_{t} h_{t}(E)(\tilde{x})+\sum_{t \in T_{E}^{-}(\bar{x})}^{s} \bar{\xi}_{t} h_{t}(E)(\tilde{x}) \\
& -\sum_{i=1}^{k} \bar{\lambda}_{i}\left(f_{i} \circ E\right)(\bar{x})-\sum_{j=1}^{m} \bar{\mu}_{j}\left(g_{j} \circ E\right)(\bar{x}) \\
& -\sum_{t \in T_{E}^{+}(\bar{x})}^{s} \bar{\xi}_{t} h_{t}(E)(\bar{x})-\sum_{t \in T_{\bar{E}}^{-}(\bar{x})}^{s} \bar{\xi}_{t} h_{t}(E(\bar{x})) \\
& \geqq\left[\sum_{I=1}^{k} \bar{\lambda}_{i} \nabla f_{i}(E(\bar{x}))+\sum_{j \in J(E(\bar{x}))} \bar{\mu}_{j} \nabla g_{j}(E(\bar{x}))\right. \\
& \left.+\sum_{t \in T_{E}^{+}(\bar{x})}^{s} \bar{\xi}_{t} \nabla h_{t}(E(\bar{x}))+\sum_{t \in T_{E}^{-}(\bar{x})}^{s} \bar{\xi}_{t} \nabla h_{t}(E(\bar{x}))\right](E(\tilde{x})-E(\bar{x})) .
\end{aligned}
$$

By the E-Karush-Kuhn-Tucker necessary optimality condition (4) and taking Lagrange multipliers $\bar{\mu}_{j}, j \notin J(\bar{x})$ and $\bar{\xi}_{t}, t \notin T_{E}^{+}(\bar{x}) \cup T_{E}^{-}(\bar{x})$, we get that the inequality:

$$
\begin{aligned}
& \sum_{I=1}^{k} \bar{\lambda}_{i}\left(f_{i} \circ E\right)(\tilde{x})+\sum_{j=1}^{m} \bar{\mu}_{j}\left(g_{j} \circ E\right)(\tilde{x}) \\
& +\sum_{t=1}^{s} \bar{\xi}_{t}\left(h_{t} \circ E\right)(\tilde{x}) \geqq \sum_{i=1}^{k} \bar{\lambda}_{i}\left(f_{i} \circ E\right)(\bar{x}) \\
& +\sum_{j=1}^{m} \bar{\mu}_{j}\left(g_{j} \circ E\right)(\bar{x})+\sum_{t=1}^{s} \bar{\xi}_{t}\left(h_{t} \circ E\right)(\bar{x})
\end{aligned}
$$

holds, contradicting (40). This completes the proof of this theorem.

From Theorem 29, it follows directly the following result.

\section{Corollary 30}

Let $\bar{x} \in \Omega_{E}$ be a (weak) Pareto solution of the $E$ vector optimization problem $\left(\mathrm{VP}_{\mathrm{E}}\right)$ and the $\mathrm{KKT}$ necessary optimality conditions (4)-(6) be fulfilled with Lagrange multiplier $\bar{\lambda} \in R^{p}, \bar{\mu} \in R^{m}$ and $\bar{\xi} \in R^{s}$. We also assume that all hypotheses of Theorem 29 are satisfied. Then $(\bar{x}, \bar{\lambda}, \bar{\mu}, \bar{\xi})$ is a vector saddle point of the vectorvalued Lagrange function $L_{p_{\bar{E}}}$ defined for $\left(\mathrm{VP}_{\mathrm{E}}\right)$.

Using the foregoing results established for the $E$-vector optimization problem $\left(\mathrm{VP}_{\mathrm{E}}\right)$, we now establish the sufficient condition for $(E(\bar{x}), \bar{\lambda}, \bar{\mu}, \bar{\xi}) \in \Omega \times R^{p} \times R^{m} \times R^{s}$ to be a vector $E$-saddle point of the Lagrange function $L_{p}$.

\section{Theorem 31}

Let $\bar{x}$ be a feasible solution of the $E$-vector optimization problem $\left(\mathrm{VP}_{\mathrm{E}}\right)$ such that $(E(\bar{x}), \bar{\lambda}, \bar{\mu}, \bar{\xi}) \in$ $\Omega \times R_{+}^{p} \times R_{+}^{m} \times R^{s}$ is an E-KKT point of the considered multicriteria optimization problem (VP). Assume, moreover, that each objective function $f_{i}, i \in I$, is $E$ convex at $\bar{x}$ on $\Omega_{E}$, each constraint function $g_{j}, j \in J$, is $E$ convex at $\bar{x}$ on $\Omega_{E}$, each constraint function $h_{t}, t \in T_{E}^{+}(\bar{x})$ and each function $-h_{t}, t \in T_{E}^{-}(\bar{x})$, are $E$-convex at $\bar{x}$ on $\Omega_{E}$. Then $(E(\bar{x}), \bar{\lambda}, \bar{\mu}, \bar{\xi}) \in \Omega \times R_{+}^{p} \times R_{+}^{m} \times R^{s}$ is a vector $E$ saddle point of the Lagrange function $L_{p}$ for (VP).

\section{Proof}

From the assumption, it follows that $\bar{x}$ is a feasible solution of the $E$-vector optimization problem $\left(\mathrm{VP}_{\mathrm{E}}\right)$ such that $(E(\bar{x}), \bar{\lambda}, \bar{\mu}, \bar{\xi}) \in \Omega \times R_{+}^{p} \times R_{+}^{m} \times R^{s}$ is an $E$-KKT point of (VP). Then, by Definition 13, the E-KKT necessary optimality conditions are fulfilled for the problem (VP) with Lagrange multipliers $\bar{\lambda}, \bar{\mu}, \bar{\xi}$. Since all assumptions of Theorem 29 are satisfied, $(\bar{x}, \bar{\lambda}, \bar{\mu}, \bar{\xi}) \in \Omega_{E} \times R_{+}^{p} \times R_{+}^{m} \times R^{s}$ is a vector saddle point of the Lagrange function $L_{E}$ defined for $\left(\mathrm{VP}_{\mathrm{E}}\right)$. This means, by Definition 24, that the following conditions:

$$
\begin{aligned}
& L_{p_{E}}(\bar{x}, \bar{\lambda}, \mu, \xi) \leqq L_{p_{E}}(\bar{x}, \bar{\lambda}, \bar{\mu}, \bar{\xi}) \forall \mu \in R_{+}^{m}, \forall \xi \in R^{s}, \\
& L_{p_{E}}(x, \bar{\lambda}, \bar{\mu}, \bar{\xi}) \pm L_{p_{E}}(\bar{x}, \bar{\lambda}, \bar{\mu}, \bar{\xi}) \forall x \in \Omega_{E}
\end{aligned}
$$

hold. By (29), (45) and (46) yield, respectively: 


$$
\begin{gathered}
\sum_{i=1}^{p} \bar{\lambda}_{i}\left(f_{i} \circ E\right)(\bar{x})+\sum_{j=1}^{m} \mu_{j}\left(g_{j} \circ E\right)(\bar{x}) \\
+\sum_{t=1}^{s} \xi_{t}\left(h_{t} \circ E\right)(\bar{x}) \leqq \sum_{I=1}^{p} \bar{\lambda}_{i}\left(f_{i} \circ E\right)(\bar{x}) \\
+\sum_{j=1}^{m} \bar{\mu}_{j}\left(g_{j} \circ E\right)(\bar{x})+\sum_{t=1}^{s} \bar{\xi}_{t}\left(h_{t} \circ E\right)(\bar{x}) \\
\forall \mu \in R_{+}^{m}, \forall \xi \in R^{s}
\end{gathered}
$$

and:

$$
\begin{gathered}
\sum_{i=1}^{p} \bar{\lambda}_{i}\left(f_{i} \circ E\right)(x)+\sum_{j=1}^{m} \bar{\mu}_{j}\left(g_{j} \circ E\right)(x) \\
+\sum_{t=1}^{s} \bar{\xi}_{t}\left(h_{t} \circ E\right)(x) \pm \sum_{I=1}^{p} \bar{\lambda}_{i}\left(f_{i} \circ E\right)(\bar{x}) \\
\sum_{j=1}^{m} \bar{\mu}_{j}\left(g_{j} \circ E\right)(\bar{x})+\sum_{t=1}^{s} \bar{\xi}_{t}\left(h_{t} \circ E\right)(\bar{x}) \forall x \in \Omega_{E} .
\end{gathered}
$$

Since $E: R^{n} \rightarrow R^{n}$ is an one-to-one and onto operator, this means that, for any $x \in \Omega_{E}$, there exists $z \in \Omega$, such that $z$ $=E(x)$. Hence, (25) and (26) yield, respectively:

$$
\begin{gathered}
\sum_{i=1}^{p} \bar{\lambda}_{i}\left(f_{i} \circ E\right)(\bar{x})+\sum_{j=1}^{m} \mu_{j}\left(g_{j} \circ E\right)(\bar{x}) \\
+\sum_{t=1}^{s} \xi_{t}\left(h_{t} \circ E\right)(\bar{x}) \leqq \sum_{i=1}^{p} \bar{\lambda}_{i}\left(f_{i} \circ E\right)(\bar{x}) \\
+\sum_{j=1}^{m} \bar{\mu}_{j}\left(g_{j} \circ E\right)(\bar{x})+\sum_{t=1}^{s} \bar{\xi}_{t}\left(h_{t} \circ E\right)(\bar{x}) \\
\forall \mu \in R_{+}^{m}, \forall \xi \in R^{s}
\end{gathered}
$$

and:

$$
\begin{gathered}
\sum_{i=1}^{p} \bar{\lambda}_{i} f_{i}(z)+\sum_{j=1}^{m} \bar{\mu}_{j} g_{j}(z)+\sum_{t=1}^{s} \bar{\xi}_{t} h_{t}(z) \pm \\
\sum_{I=1}^{p} \bar{\lambda}_{i}\left(f_{i} \circ E\right)(\bar{x})+\sum_{j=1}^{m} \bar{\mu}_{j}\left(g_{j} \circ E\right)(\bar{x}) \\
+\sum_{t=1}^{s} \bar{\xi}_{t}\left(h_{t} \circ E\right)(\bar{x}) \forall z \in \Omega .
\end{gathered}
$$

By Definition 25, (49) and (50) imply that $(E(\bar{x}), \bar{\lambda}, \bar{\mu}, \bar{\xi}) \in \Omega \times R_{+}^{p} \times R_{+}^{m} \times R^{s} \quad$ is a vector $E$-saddle point of the vector-valued Lagrange function $L_{p}$ in (VP).

\section{Corollary 32}

Let $\bar{x} \in \Omega$ be a (weak) $E$-Pareto solution of the $E$ vector optimization problem $\left(\mathrm{VP}_{\mathrm{E}}\right)$ and, thus, $E(\bar{x})$ be a (weak) E-Pareto solution of the considered multiobjective programming problem (VP). Further, assume that the $E$-KKT conditions (4)-(6) are satisfied at $E(\bar{x})$ with Lagrange multiplier $\bar{\lambda} \in R^{p}, \bar{\mu} \in R^{m}$ and $\bar{\xi} \in R^{s}$. If all assumptions of Theorem 29 are satisfied, then $(E(\bar{x}), \bar{\lambda}, \bar{\mu}, \bar{\xi})$ is a vector $E$-saddle point of the vector-valued Lagrangian $L_{p}$ in (VP).

As it follows from the above proofs, the definition of a vector $E$-saddle point of the vector-valued Lagrange function $L_{p}$ can be formulated as follows.

\section{Definition 33}

Let $\bar{x}$ be a feasible solution of the $E$-vector optimization problem $\left(\operatorname{VP}_{\mathrm{E}}\right)$. A point $(E(\bar{x}), \bar{\lambda}, \bar{\mu}, \bar{\xi}) \in$ $\Omega \times R_{+}^{p} \times R_{+}^{m} \times R^{s}$ is said to be an $E$-saddle point for the considered multiobjective programming problem (VP) if:

i. $\quad L_{p}(E(\bar{x}), \bar{\lambda}, \mu, \xi) \leqq L_{p}(E(\bar{x}), \bar{\lambda}, \bar{\mu}, \bar{\xi}) \forall \mu \in R_{+}^{m}, \forall \xi \in R^{s}$,

ii. $\quad L_{p}(E(x), \bar{\lambda}, \bar{\mu}, \bar{\xi}) \nless L_{p}(E(\bar{x}), \bar{\lambda}, \bar{\mu}, \bar{\xi}) \forall E(x) \in \Omega$.

Now, we illustrate the main result established in this section by an example of an E-differentiable vector optimization problem.

\section{Example 34}

Consider the following nondifferentiable vector optimization problem:

$$
\begin{gathered}
\text { minimize } f\left(x_{1}, x_{2}\right)=\left(2 x_{1}+2 \sqrt[3]{x_{1}}-x_{2}, x_{2}^{2}+x_{2}-\sqrt[3]{x_{1}}\right) \\
\text { s.t. } g\left(x_{1}, x_{2}\right)=\sqrt[3]{x_{1}^{2}}-x_{2} \leqq 0 \quad(V P 2) \\
h\left(x_{1}, x_{2}\right)=x_{2}^{2}-\sqrt[3]{x_{1}}=0
\end{gathered}
$$

Note that $\Omega=\left\{\left(x_{1}, x_{2}\right) \in R^{2}: \sqrt[3]{x_{1}^{2}}-x_{2} \leqq 0 \wedge x_{2}^{2}-\sqrt[3]{x_{1}}=0\right\}$. Let $E: R^{2} \rightarrow R^{2}$ be an operator defined by $E\left(x_{1}, x_{2}\right)=\left(x_{1}^{3}, x_{2}\right)$. For the considered vector optimization problem (VP2), we define its associated Emulticriteria optimization problem $\left(\mathrm{VP} 2_{\mathrm{E}}\right)$ as follows:

$$
\begin{gathered}
\text { minimize } f\left(E\left(x_{1}, x_{2}\right)\right)=\left(2 x_{1}^{3}+2 x_{1}-x_{2}, x_{2}^{2}+x_{2}-x_{1}\right) \\
\text { s.t. } g\left(E\left(x_{1}, x_{2}\right)\right)=x_{1}^{2}-x_{2} \leqq 0 \quad\left(V P 2_{E}\right) \\
h\left(E\left(x_{1}, x_{2}\right)\right)=x_{2}^{2}-x_{1}=0 .
\end{gathered}
$$

Then, $\quad \Omega_{E}=\left\{\left(x_{1}, x_{2}\right) \in R^{2}: x_{1}^{2}-x_{2} \leqq 0 \wedge x_{2}^{2}-x_{1}=0\right\} \quad$ and $\bar{x}=(0,0)$ is a feasible solution. Further, note that all functions constituting the considered vector optimization problem (VP2) are $E$-differentiable at $\bar{x}=(0,0)$. Then, it can also be proved that the $E$-KKT necessary optimality conditions (4)-(6) are fulfilled at $\bar{x}=(0,0)$ with Lagrange multipliers $\bar{\lambda}_{1}=1, \bar{\lambda}_{2}=\frac{3}{2}, \bar{\mu}=\frac{1}{2}$ and $\bar{\xi}=\frac{1}{2}$. Since all assumptions of Theorem 29 are fulfilled, $(\bar{x}, \bar{\lambda}, \bar{\mu}, \bar{\xi})$ is a saddle point of the vector-valued Lagrange function $L_{p_{E}}$ defined for the E-multicriteria optimization problem 
$\left(\mathrm{VP} 2_{\mathrm{E}}\right)$. Furthermore, by Theorem 31, it follows that $(E(\bar{x}), \bar{\lambda}, \bar{\mu}, \bar{\xi})$ is an $E$-saddle point of the Lagrange function $L_{p}$ of the vector optimization problem (VP2).

\section{Conclusion}

In this paper, to the best of our knowledge, for the first time, the saddle point criteria have been derived for the class of (not necessarily) differentiable multiobjective programming problems with both inequality and equality constraints in which the involved functions are $E$-differentiable. For the considered $E$ differentiable multiobjective programming problem, its associated $E$-vector optimization problem has been constructed in which the involved functions are differentiable in the usual sense. Further, two types of the so-called $E$-saddle point criteria have been derived for the original (not necessarily) differentiable multiobjective programming problem using the similar results established under E-convexity hypotheses for its associated $E$-vector optimization problem in which the involved functions are differentiable in the usual sense. The $E$-saddle point criteria presented in the paper have been illustrated by examples of nondifferentiable multiobjective programming problems with $E$-differentiable functions. This kind of studies seems to be important because the methods used to characterize saddle point criteria for (not necessarily) differentiable multicriteria optimization problems are based on the similar results established for differentiable vector optimization problems.

The results established in this paper warrant further research in several directions. In particular, it would be of great interest to examine how the results of this paper can be generalized and extended for other classes E-differentiable multiobjective programming problems. This may be the topic of some of our forthcoming papers.

\section{Acknowledgment}

The authors thank the Editor and the Reviewers for their helpful comments, which certainly improved the presentation of the content of the paper.

\section{Author's Contributions}

Both authors participated in all aspects of the research and the writing of the article.

\section{Ethics}

The authors confirm that this article is an original work and contains unpublished material. The authors declare no conflict of interest and there are no ethical issues involved.

\section{References}

Adán, M. and V. Novo, 2005. Duality and saddle-points for convex-like vector optimization problems on real linear spaces. Top, 13: 343-357. DOI: $10.1007 / \mathrm{BF} 02579060$

Antczak, T., 2003. A new approach to multiobjective programming with a modified objective function. J. Global Optim., 27: 485-495.

DOI: $10.1023 / \mathrm{A}: 1026080604790$

Antczak, T., 2005. Saddle point criteria and duality in multiobjective programming via an $\eta$-approximation method. ANZIAM J., 47: 155-172.

DOI: $10.1017 / \mathrm{S} 1446181100009962$

Antczak, T., 2015. Saddle point criteria and Wolfe duality in nonsmooth $(\Phi, \rho)$-invex vector optimization problems with inequality and equality constraints. Int. J. Comput. Math., 92: 882-907. DOI: $10.1080 / 00207160.2014 .925191$

Antczak, T. and N. Abdulaleem, 2018. E-optimality conditions and Wolfe E-duality for E-differentiable vector optimization problems with inequality and equality constraints. J. Nonlinear Sci. Appl.

Bhatia, G., 2008. Optimality and mixed saddle point criteria in multiobjective optimization. J. Math. Anal. Applic., 342: 135-145.

DOI: $10.1016 / \mathrm{j}$.jmaa.2007.11.042

Bigi, G., 2001. Saddle Point Optimality Criteria in Vector Optimization. In: Optimization in Economics, Finance and Industry, Crespi, G.P., G. Giorgi, A. Guerraggio, D. La Torre and E. Miglierina et al. (Eds.), Datanova, Milano, pp: 85-102.

Craven, B.D., 1990. Quasimin and quasisaddlepoint for vector optimization. Numer. Func. Anal. Opt., 11: 45-54. DOI: 10.1080/01630569008816360

Ehrgott, M. and M.M. Wiecek, 2005. Saddle points and Pareto points in multiple objective programming. J. Optim. Theory Applic., 32: 11-33.

Jiang, J. and S. Xu, 2010. Optimality and saddle point for vector optimization under semilocally Bpreinvex. Proceedings of the International Conference on Artificial Intelligence and Computational Intelligence, Oct. 23-24, IEEE Xplore Press, Sanya, China, pp: 382-386.

DOI: 10.1109/AICI.2010.317

Kuk, H., G.M. Lee and D.S. Kim, 1998. Nonsmooth multiobjective programs with $\mathrm{V}-\rho$-invexity. Indian J. Pure Applied Math., 29: 405-412.

Kumar, A. and P.K. Garg, 2015. Mixed saddle point and its equivalence with an efficient solution under

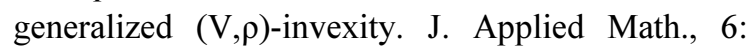
1630-1637. DOI: 10.4236/am.2015.69145

Maciel, M.C., S.A. Santos and G.N. Sottosanto, 2016. On the Fritz John saddle point problem for differentiable multiobjective optimization. Opsearch, 53: 917-933. DOI: $10.1007 / \mathrm{s} 12597-016-0253-\mathrm{x}$ 
Megahed, A.A., H.G. Gomma, E.A. Youness and A.H. El-Banna, 2013. Optimality conditions of E-convex programming for an $E$-differentiable function. J. Inequal. Appl., 2013: 246-246.

DOI: $10.1186 / 1029-242 X-2013-246$

Mishra, S.K. and G. Giorgi, 2008. Invexity and Optimization (Nonconvex Optimization and its Applications). 1st Edn., Springer, ISBN-10: 3540785612, pp: 266.

Li, Z.F. and S.Y. Wang, 1994. Lagrange multipliers and saddle points in multiobjective programming. J. Optim. Theory Applic., 83: 63-81. DOI: 10.1007/BF02191762

Van Rooyen, M., X. Zhou and S. Zlobec, 1994. A saddlepoint characterization of Pareto optima. Math. Program., 67: 77-88. DOI: 10.1007/BF01582213

Tanaka, T., 1990. A characterization of generalized saddle points for vector-valued functions via scalarization. Nihonkai Math. J., 209-227.

Tanaka, T., 1994. Generalized quasiconvexities, cone saddle points and minimax theorem for vectorvalued functions. J. Optim. Theory Applic., 81: 355-377. DOI: 10.1007/BF02191669
Tanino, T., 1982. Saddle points and duality in multiobjective programming. Int. J. Syst. Sci., 13: 323-335. DOI: $10.1080 / 00207728208926352$

Vályi, I., 1987. Approximate saddle-point theorems in vector optimization. J. Optim. Theory Applic., 55: 435-448. DOI: 10.1007/BF00941179

Varalakshmi, G., P. Chandrakala and P.R.S. Reddy, 2010. Weak vector saddle point theorem under V- $\rho$-invexity. Bull. Pure Applied Sci. Math., 29: 453-465.

Yan, Z. and S. Li, 2004. Saddle point and generalized convex duality for multiobjective programming. J. Applied Math. Comput., 15: 227-235. DOI: $10.1007 / \mathrm{BF} 02935757$

Youness, E.A., 1999. E-convex sets, E-convex functions and $E$-convex programming. J. Optim. Theory Applic., 102: 439-450. DOI: $10.1023 / \mathrm{A}: 1021792726715$

Zeng, R., 2017. Saddle-point theorems for the generalized cone-convex optimizations of set-valued functions. arXiv preprint arXiv. 